\title{
Phase controlled superconducting proximity effect probed by tunneling spectroscopy
}

\author{
H. le Sueur, P. Joyez, H. Pothier, C. Urbina, and D. Esteve \\ Quantronics group, Service de Physique de l'Etat Condensé (CNRS URA 2464), CEA-Saclay, 91191 Gif-sur-Yvette, France
}

(Dated: August 12, 2021)

\begin{abstract}
Using a dual-mode STM-AFM microscope operating below $50 \mathrm{mK}$ we measured the Local Density of States (LDoS) along small normal wires connected at both ends to superconductors with different phases. We observe that a uniform minigap can develop in the whole normal wire and in the superconductors near the interfaces. The minigap depends periodically on the phase difference. The quasiclassical theory of superconductivity applied to a simplified 1D model geometry accounts well for the data.
\end{abstract}

PACS numbers: 74.45.+c, 07.79.-v, 74.78.Na, 73.20.At

The proximity effect at the interface between a normal metal $(\mathrm{N})$ and a superconductor $(\mathrm{S})$ consists in the weakening of electron pair correlations on the $\mathrm{S}$ side and in their appearance on the $\mathrm{N}$ side, which can then acquire superconductor-like properties (for a review, see [1]). In the case of diffusive metals, the quasiclassical theory of superconductivity [2] provides a powerful framework to describe this proximity effect. It predicts that if the $\mathrm{N}$ region is smaller than the electron phase coherence length, it then behaves as a genuine superconductor, i.e. it can carry a supercurrent and there is an energy range $[-\delta,+\delta]$ around the Fermi energy in which there are no available states for quasiparticles. As $\delta$ is smaller than the gap $\Delta$ of the bulk superconductor, it has been dubbed the "minigap" [2]. A remarkable feature is that this minigap is the same everywhere in the structure, i.e. both in the $\mathrm{N}$ and in the $\mathrm{S}$ electrodes. In particular, this implies a non-zero Local Density of States (LDoS) in the superconductor between $\delta$ and $\Delta$. This additional density of states, which can be seen as the evanescent tail of normal electrons undergoing Andreev reflection [1] at the NS interface, dies exponentially when moving into the superconductor. These predictions are illustrated in Fig. 1a for the case of a one dimensional SNS structure in which $\delta$ is furthermore predicted to depend periodically on the phase difference $\varphi$ between the order parameters of the two superconductors [3] and vanishes at $\varphi=\pi \bmod 2 \pi$, as shown in Fig. 1b. Although spectral properties of proximity structures have been partially probed in spatial measurements of the $\operatorname{LDoS}[4,5,7,6,7,8]$ and in transport measurements (refs. in [1] and [9, 10, 11, 12]), neither the existence of a uniform minigap nor its phase modulation have been reported. In this Letter we present high resolution measurements of the phase and space dependence of the LDoS on both sides of the interfaces of SNS structures, and compare them to the theoretical predictions.

In a Scanning Tunneling Microscope (STM), the dependence of the tunneling current $I$ on the tip-to-sample voltage $V$ directly probes the electronic states available at energy $\mathrm{eV}$ in the electrode just underneath the sharp tip. Variations of the LDoS over small distances can therefore be accessed taking advantage of the high spatial

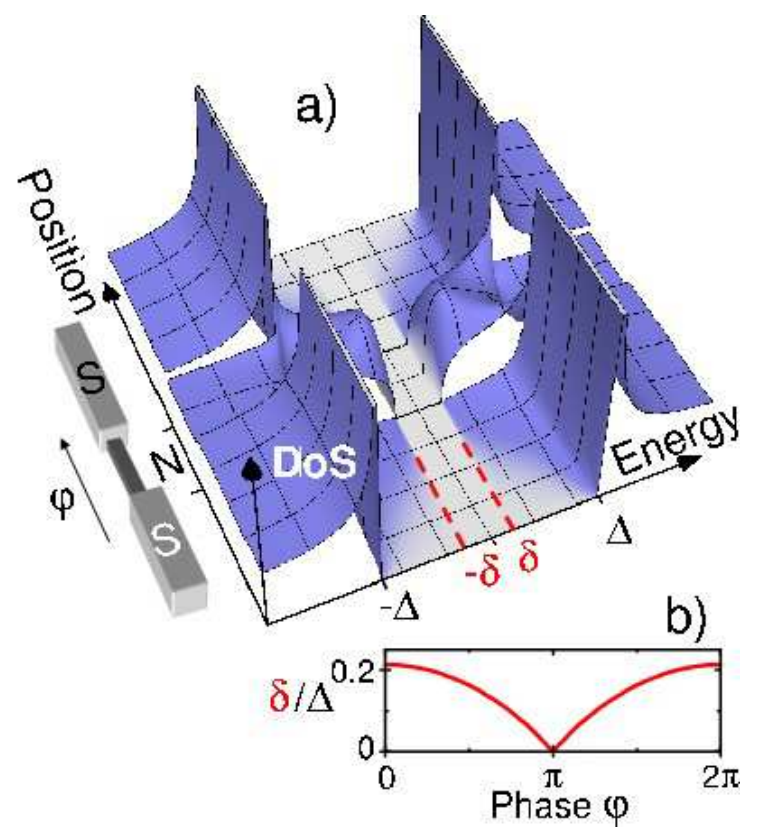

FIG. 1: (color online) (a) LDoS in a 1-D SNS structure, obtained by solving the Usadel equations of the quasiclassical theory of superconductivity in diffusive metals, taking into account scattering at interfaces and geometrical parameters [15]. $\Delta$ is the gap of the superconductor. A uniform minigap $\delta$, defining a range of energy $2 \delta$ in which the LDOS is exactly zero, appears across the normal region and in the $\mathrm{S}$ electrodes. In the latter, the finite conductance between $\delta$ and $\Delta$ decreases exponentially away from the interfaces, and the BCS DoS is eventually recovered. The color scale was chosen to enhance the visibility of the minigap. (b) Predicted $2 \pi$-periodic phase modulation of $\delta$.

resolution of the STM. In a measurement with a normal metal tip, the differential conductance $d I / d V(V)$ at small energies is simply proportional to the LDoS of the electrode, broadened by an instrumental function which is ideally the derivative of the Fermi distribution function of the electrons in the tip at temperature $T_{e}$ [13]. This sets a limit to the energy resolution of such tunneling spectroscopy at about $3.5 k_{B} T_{e}$, which can be nevertheless small enough to resolve the features due to the prox- 

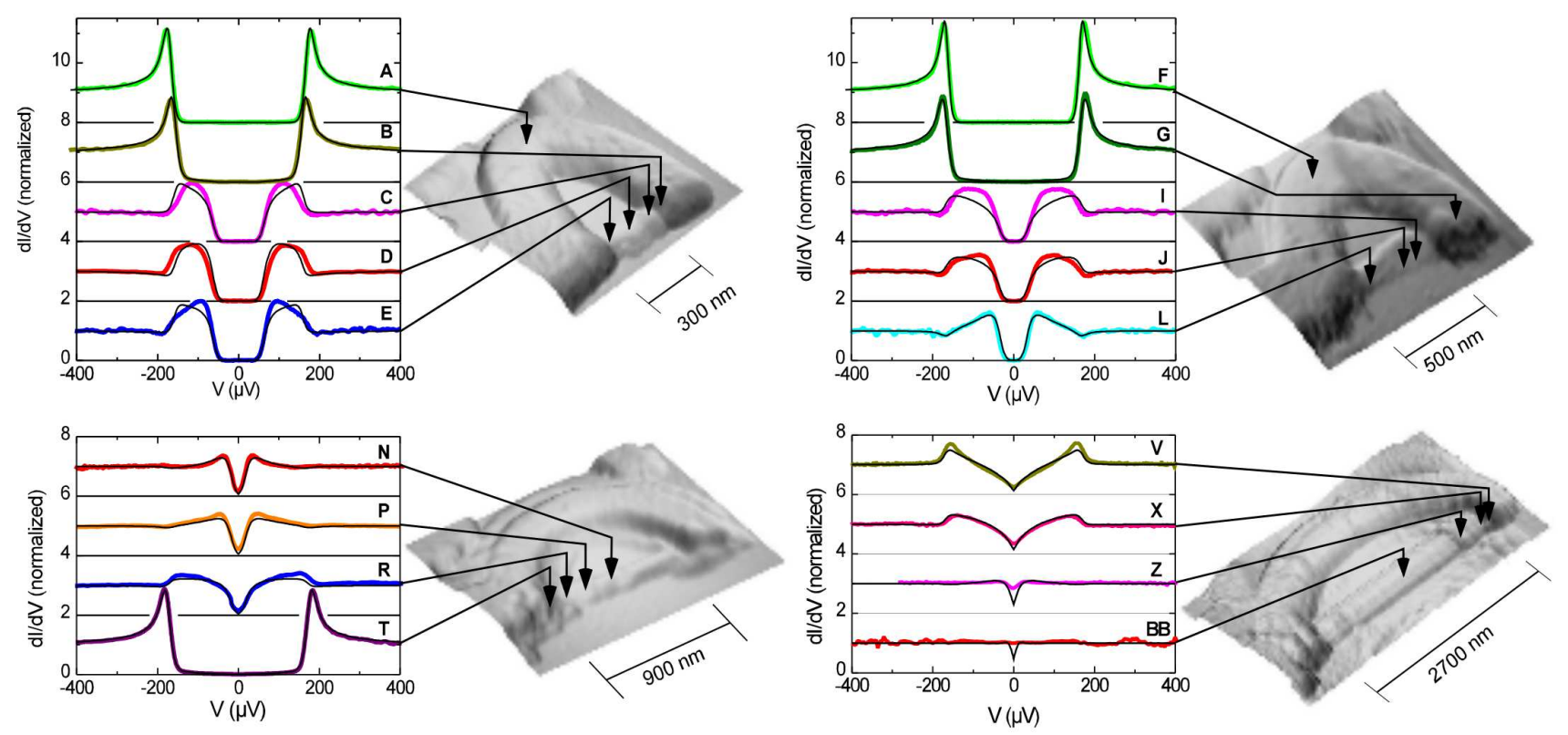

FIG. 2: (color online) Measured (thick grayed lines) differential conductance taken for zero flux through the loop at various positions (indicated by the arrows and labeled by capital letters) of the four SNS structures shown by the AFM images (taken at $35 \mathrm{mK}$ ). The length of the silver wire is respectively (a) $300 \mathrm{~nm}$, (b) $500 \mathrm{~nm}$, (c) $900 \mathrm{~nm}$, (d) $2700 \mathrm{~nm}$. The curves are normalized to 1 at large voltage and are shifted by integer numbers for clarity. The thin black solid lines correspond to the model based on the quasiclassical theory of superconductivity introduced in the text and described more in depth in Ref. [15].

imity effect, provided the electrons in the tip are cooled well below the temperature $\delta / k_{B}$.

In order to perform such measurements on nanocircuits, which contain large insulating areas on which STMs cannot be operated, we have designed and built a cryogenic dual mode STM-AFM (Atomic Force Microscope) [14, 15], operating with a single metallic tip. This local probe sensor consists of an electrochemically sharpened tungsten wire [16] glued on one prong of a miniature piezoelectric quartz tuning fork. The latter is a high quality factor electromechanical resonator, which here serves as the AFM transducer [17]. Other dual mode instruments are being developed and used elsewhere [18, 19]. The AFM mode is used to acquire detailed topographic images of the samples which later on allow for accurate positioning of the tip for STM spectroscopy. The microscope is mounted in a table-top dilution refrigerator with a base temperature of $\sim 35 \mathrm{mK}$. By itself this is not sufficient to ensure a low electronic temperature $T_{e}$ and therefore a high energy resolution: it is of critical importance to also shield and filter all the electrical lines needed to operate the microscope, and to measure the tunnel current with a low back-action amplifier. For these purposes, we developed microfabricated cryogenic filters [20] and a custom low-noise, differential-sensing current amplifier [21]. After cooling down, the tip is moved on the sample substrate towards the structures of interest, using a 3-axes capacitance-indexed coarse-positioning sys- tem based on stick-slip motion. During this stage, the microscope is operated in AFM mode, and recognition of superficial patterns etched in the substrate is used to locate the structures. Once a satisfactory AFM image is recorded spectroscopy measurements are performed in STM mode at several positions along the mapped structure.

AFM pictures of four of the SNS structures that were measured are shown in Fig. 2. They consist of a loop formed with a nominally $60 \mathrm{~nm}$-thick, $200 \mathrm{~nm}$-wide Ushaped superconducting aluminum $(\mathrm{Al})$ electrode and a nominally $50 \mathrm{~nm}$-wide and $30 \mathrm{~nm}$-thick normal silver (Ag) wire. The Al film is designed to overlap the $\mathrm{Ag}$ wire at both ends over nominally $50 \mathrm{~nm}$. Several structures were fabricated simultaneously on the same insulating substrate chip with $\mathrm{Ag}$ wire lengths ranging from 300 $\mathrm{nm}$ to $3 \mu \mathrm{m}$ (for details on the fabrication see [14, 15]). The aspect ratio of the structures was chosen such that a magnetic flux $\Phi$ threading the loop imposes a phase difference $\varphi \simeq 2 \pi \Phi / \Phi_{0}$ across the silver wire [22], with $\Phi_{0}=h / 2 e$ the flux quantum. To sink the tunnel current when performing spectroscopy, the $\mathrm{Al}$ electrode is connected to ground on one side. The visible part of the $\mathrm{Ag}$ wires is covered by a fully oxidized $2 \mathrm{~nm}$-thick aluminum film. By putting the tip in mechanical contact with this oxide layer, one can obtain nearly drift-free tunnel contacts with conductance of the order of $1 \mu \mathrm{S}$. This allows to take reproducible, high resolution spectra, by measur- 
ing the differential conductance as a function of the DC tip-sample bias voltage, using a lock-in amplifier with typically a $1 \mu \mathrm{V}$ AC excitation.

When the tip is placed on top of an $\mathrm{Al}$ electrode far away from any contact to the Ag, the differential conductance (Fig. 2a curve A, and Fig. 2b curve F) displays the well known behavior of a Bardeen-CooperSchrieffer (BCS) superconductor, with an energy gap and its characteristic singularity at the gap edge. These curves are well fitted with a BCS DoS, with an energy gap $\Delta=170 \mu \mathrm{eV}$ for $\mathrm{Al}$, and an effective electronic temperature $T_{e}=65 \mathrm{mK}$. This is higher than the temperature of the refrigerator during the measurements $(35 \mathrm{mK})$, probably because of a still insufficient reduction of the electrical noise. The corresponding energy resolution of $20 \mu \mathrm{eV}$ is nevertheless, to our knowledge, the best obtained so far in STM spectroscopy.

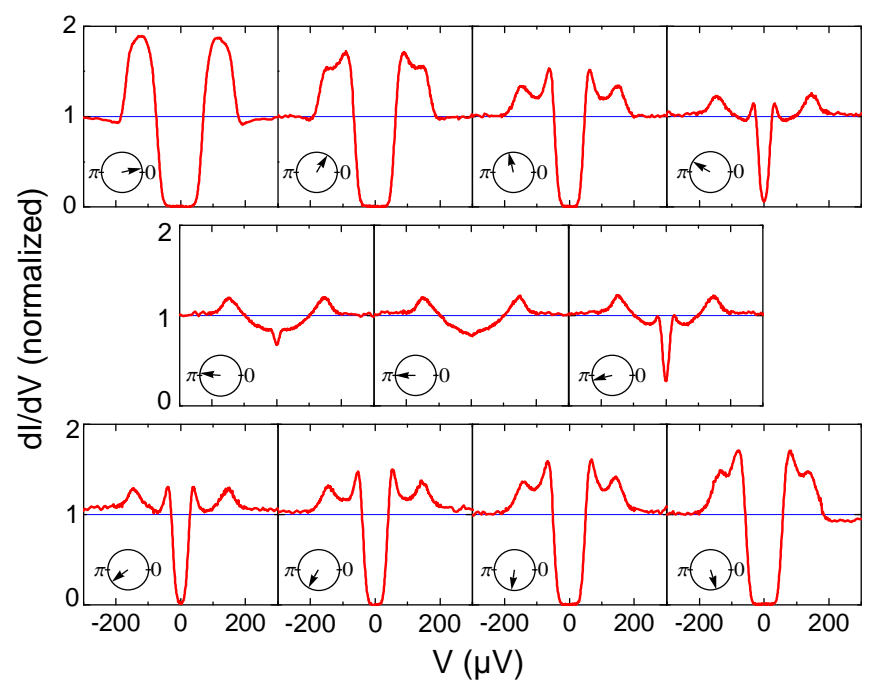

FIG. 3: Differential conductance $d I / d V(V)$ versus voltage measured in the middle of the $300 \mathrm{~nm}$-long Ag wire (position $\mathrm{D}$ ) for different values of the phase difference across it as shown by the phase-clocks $(\varphi / \pi=0.06,0.32,0.57,0.83 ; 0.96$, $1.00,1.08 ; 1.21,1.34,1.46,1.59)$.

In Fig. 2 we also show the LDoS measured at zero flux for several positions along each of the four normal wires. Both in the 300 and in the $500 \mathrm{~nm}$-long $\mathrm{Ag}$ wire, the differential conductance vanishes around zero voltage, on an energy range independent of position, which is the signature of a minigap in the DoS. The minigap is larger for the shorter wire. In the $900 \mathrm{~nm}$-long wire, the conductance is strongly reduced on a smaller energy range around zero, but remains always finite. Finally, in the middle of the $2.7 \mu \mathrm{m}$-long wire, $d I / d V(V)$ is nearly constant, i.e. unaffected by the proximity effect. Yet, in this long wire, the LDoS is strongly affected near the ends of the wire. When the tip is placed on the $\mathrm{Al}$ near a contact to the $\mathrm{Ag}$ wire, a small finite conductance is observed at subgap voltages (see e.g. curve $\mathrm{G}$ in Fig. $2 \mathrm{~b}$, curve $\mathrm{T}$

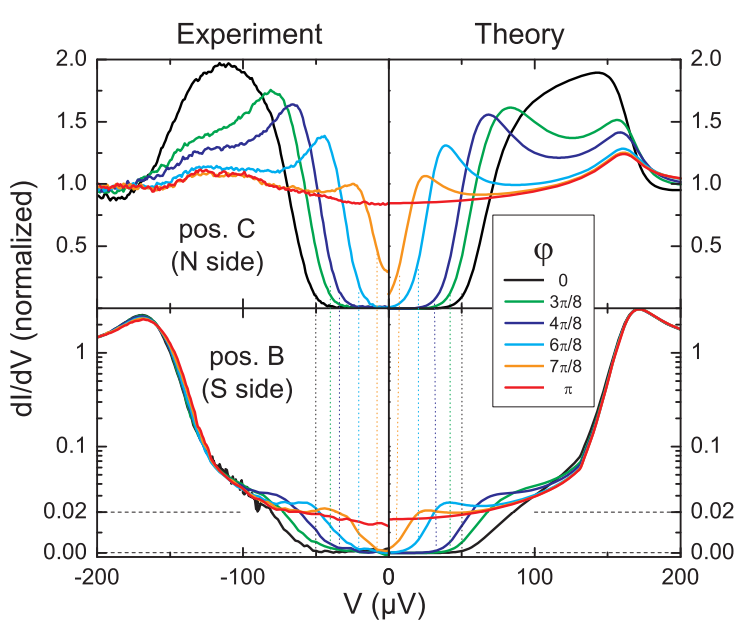

FIG. 4: (color online) Differential conductance versus voltage, for different values of the phase difference $\varphi$ across the $300 \mathrm{~nm}$ long Ag wire. Left quadrants : measured; Right quadrants : calculated. Top : on the $\mathrm{N}$ side (position C); Bottom : on the $\mathrm{S}$ side, close to the interface (position B - Note that the scale is linear up to 0.02 and logarithmic above, to magnify the variation for small subgap conductance). This shows that the minigap is also present on the superconductor side with the same value as on the $\mathrm{N}$ side. Dashed lines are guides to the eye.

in Fig. 2c and curve B in Fig. 2a and the corresponding close-up in the bottom part of Fig. 4). In structures displaying a finite minigap in the $\mathrm{N}$ wire we find that the same minigap is also present on the $\mathrm{S}$ side, despite very different overall shapes of the LDoS. Correlatively, the order parameter is slightly reduced close to the interface as shown by a shift in the position of the peak in the density of states $(160 \mu \mathrm{V}$ at position $\mathrm{B} v s .170 \mu \mathrm{V}$ at position A in Fig. 2a). Farther from the normal metal (positions A and F in Fig. 2), the subgap LDoS vanishes.

The variations with the phase difference $\varphi$ of the LDoS in the middle of the shortest wire (position D) are shown in Fig. 3. As $\varphi$ is increased, $d I / d V(V)$ gradually deforms and $\delta$ disminishes. Around $\varphi=\pi$, the minigap vanishes, and the LDoS approaches that of the normal state: superconductivity is then maximally frustrated in the wire. This behavior is $\Phi_{0}$-periodic with the applied flux [15], which provides the phase calibration for each structure. The left quadrants of Fig. 4 show the measured variations of the subgap conductance on both the $\mathrm{S}$ and $\mathrm{N}$ side (positions B and C respectively), establishing further that for all values of $\varphi$, the minigap is the same in both the $\mathrm{S}$ and $\mathrm{N}$ part of the structure. A similar phase modulation is observed in the 500 and $900 \mathrm{~nm}$-long wires.

The above measurements can be compared with the predictions of the quasiclassical theory of superconduc- 
tivity in diffusive metals. This theory is based on the evaluation of the quasiclassical retarded Green function which, for a superconductor, is a $2 \times 2$ matrix of disorder-averaged correlation functions in electron-hole (or Nambu) space. This matrix depends on position and energy and obeys a diffusion equation, known as the Usadel equation, with boundary conditions at the interfaces 23]. Finding the LDoS in our structures requires, in principle, to specify their full 3-D geometry (including the interfaces and their properties), a complex task which, as far as we know, has never been done. For simplicity, we restrict here to solving a crude onedimensional SNS model involving geometrical and material parameters common to all structures (as they are fabricated simultaneously), and per-structure parameters characterizing the NS interfaces [15]. Due to the finite extent of the overlap interfaces of $\mathrm{N}$ and $\mathrm{S}$ in the samples, one cannot define exactly the tip-to-interface distance. Hence, for simplicity, in the model the effective position of the tip along the effective $\mathrm{N}$ wire length is crudely mapped to the apparent position of the tip on the AFM image, with an offset roughly equal to half the overlap length (see [15] for further details). Finally, in order to account for the effective temperature of the tip, we compare the measurements with the outcome of this model convolved with the derivative of Fermi function at $65 \mathrm{mK}$. In spite of the simplifying assumptions of the model, an overall agreement is obtained for the phase, space, wire length and energy dependence of all the conductance curves, as shown in Figures 2 and 4. Deviations are most pronounced in the shortest wire at positions near the wire ends (e.g. positions $\mathrm{C}$ and $\mathrm{E}$ ), for which the $1 \mathrm{D}$ approximation neglecting the finite extent of the overlap junction geometry is relatively more important. Also, a narrow dip in the conductance predicted near $V=0$ at the middle of the $2.7 \mu \mathrm{m}$-long wire is not observed (see Fig. 2d), which may point to some uncontrolled depairing mechanism not taken into account in the model.

In conclusion, we have measured with unprecedented energy resolution the spatial and phase dependence of the superconducting proximity effect in a series of diffusive structures. In particular, we have shown that an identical phase-dependent minigap is present in both the $\mathrm{N}$ and $\mathrm{S}$ electrodes. This works complements our understanding of the proximity effect and supports its description by the quasiclassical theory. These findings are a first illustration of what can be achieved with this low temperature AFM-STM. There is a large variety of hybrid nanocircuits, which combine for example magnetic materials, semiconductors or even molecules such as carbon nanotubes, that should also give rise to a number of interesting short range "proximity effects" at interfaces. In addition, the capabilities of this new instrument can be extended in various ways. For instance, a superconducting tip would allow probing the superconducting condensate [24] or the electronic distribution functions in nanocircuits driven out-of-equilibrium, providing insight on local electron dynamics in the samples 25]. One could also use spin-polarized tips [26] to resolve spin order, or use the sensor for local gating or electrostatic force measurements [27] on the circuit. This combined AFM-STM cryogenic microscope is thus a versatile tool that opens broad perspectives in mesoscopic physics.

We gratefully acknowledge technical support by P.-F. Orfila and P. Senat and help from the whole Quantronics group. We are also indebted to N. Agraï, H. Bouchiat, C. Chapelier, H. Courtois, S. Guéron, T. Heikkilä, G. Rubio-Bollinger, E. Scheer, H. Suderow and Y. de Wilde for useful exchanges. We particularly thank J.C. Cuevas for guidance on theory and numerics. We acknowledge support by ANR-07-Blan-0240-01 and AC Nano 2003 NR110.

[1] B. Pannetier and H. Courtois, J. Low Temp. Phys. 118, 599 (2000).

[2] W. Belzig et al., Superlatt. and Microstruct. 25, 1251 (1999).

[3] F. Zhou et al., J. Low Temp. Phys. 110, 841 (1998).

[4] S. Guéron et al., Phys. Rev. Lett. 77, 3025 (1996).

[5] M. Vinet, C. Chapelier, and F. Lefloch, Phys. Rev. B 63, 165420 (2001).

[6] N. Moussy, H. Courtois, and B. Pannetier, Europhys. Lett. 55, 861 (2001).

[7] A.K. Gupta et al., Phys. Rev. B 69, 104514 (2004).

[8] W. Escoffier et al., Phys. Rev. Lett. 93, 217005 (2004).

[9] P. Dubos et al., Phys. Rev. Lett. 87, 206801 (2001).

[10] V.T. Petrashov et al., Phys. Rev. Lett. 95, 147001 (2005).

[11] M. S. Crosser et al., Phys. Rev. Lett. 96, 167004 (2006).

[12] L. Angers et al., arXiv:0708.0205 (2007).

[13] O. Fischer et al., Rev. Mod. Phys. 79, 353 (2007).

[14] H. le Sueur, Ph.D. Thesis (in English), Université Paris 6, 2007. Available online at http://tel.archives-ouvertes.fr/tel-00261434/en/

[15] See EPAPS Document No. E-PRLTAO-??-????? for additional data, as well as experimental and modeling details (http://www.aip.org/pubservs/epaps.html).

[16] M. Kulawik et al., Rev. Sci. Instrum. 74, 1027 (2003).

[17] K. Karrai and R. D. Grober, Appl. Phys. Lett. 66, 1842 (1995).

[18] J. Senzier, P.S. Luo, and H. Courtois, Appl. Phys. Lett. 90, 043114 (2007).

[19] R. H. M. Smit et al., Rev. Sci. Instrum. 78, 113705 (2007).

[20] H. le Sueur and P. Joyez, Rev. Sci. Instrum. 77, 115102 (2006).

[21] H. le Sueur and P. Joyez, Rev. Sci. Instrum. 77, 123701 (2006).

[22] Within correcting factors of the order $\left(L_{S}+L_{G}\right) / L_{N}$, where $L_{N}$ and $L_{S}$ are the kinetic inductance of respectively the normal and the superconducting wires and $L_{G}$ is the geometrical inductance of the complete loop. For our samples these corrections are always smaller than $10 \%$. See [14, 15] for details. 
[23] Yu.V. Nazarov, Superlatt. and Microstruct. 25, 1221 (1999).

[24] Th. Proslier et al., Europhys. Lett. 73, 962 (2006).

[25] H. Pothier et al., Phys. Rev. Lett. 79, 3490 (1997).

[26] M. Bode and R. Wiesendanger, in Magnetic Microscopy of Nanostructures, H. Hopster and H. P. Oepen, Eds, Springer (Berlin, Heidelberg) (2005).

[27] M. T. Woodside and P. L. McEuen, Science 296, 1098 (2002). 